\title{
SPECTRUM OF FREQUENCY MODULATION OF SERPENTINE EMISSION AS A REFLECTION OF THE SOLAR FLUCTUATION SPECTRUM
}

\author{
B.V. Dovbnya' ${ }^{1}$ B.I. Klain ${ }^{1}$, A.V. Guglielmi ${ }^{2}$, A.S. Potapov ${ }^{3}$ \\ ${ }^{1}$ Borok Geophysical Observatory of IPE RAS, Borok, Russia \\ ${ }^{2}$ Schmidt Institute of Physics of the Earth, RAS, Moscow, Russia \\ ${ }^{3}$ Institute of Solar-Terrestrial Physics SB RAS, Irkutsk, Russia \\ dovbnya@inbox.ru,klain@borok.yar.ru,guglielmi@mail.ru,potapov@iszf.rrk.ru
}

We study frequency modulation of serpentine emission (SE), using data from the Vostok Antarctic station. It is shown that the previously observed 5-minute modulation of the SE carrier frequency is the most prominent and stable in the emission spectrum. Frequency fluctuations of this period are present in about $70 \%$ of the total SE observation time under moderately quiet geomagnetic conditions $\left(K_{p}=0-2\right)$. We performed a per-pixel processing of $S E$ dynamic spectra and found that the power spectrum of the signal frequency modulation contains a clearly visible peak at periods close to 5 minutes. A detailed study shows the emission spectrum matching the frequency range of the solar photospheric oscillations. The results of the analysis allow us to conclude that the 5-minute modulation of the SE carrier frequency can be viewed as a reflection of photospheric fluctuations with the same period that is typical for the solar eigenoscillations.

Keywords: Serpentine emission $\cdot$ Frequency modulation $\cdot$ Power spectrum $\cdot$ Photospheric oscillations

\section{INTRODUCTION}

Serpentine emission is quasi-continuous geomagnetic pulsations, which are observed for hours and sometimes for days in the polar cap under quiet and moderate geomagnetic conditions. The main property of this emission is determined by deep modulation of the carrier frequency changing by 1-2 octaves in a frequency range from $0.01 \mathrm{~Hz}$ to $5 \mathrm{~Hz}$. The emission was discovered in Antarctica at the station Vostok in the 1970s [Guglielmi, Dovbnya, 1973, 1974a,b]. Characteristic properties of SE allowed us to assume that the frequency-modulated oscillations penetrate into polar caps from the interplanetary medium, where they are excited in the form of ion-cyclotron waves due to plasma instability with anisotropic velocity distribution of ions [Guglielmi, Dovbnya, 1973, 1974a,b]. Then, SE was also recorded at the station Davis [Morris, Cole, 1987] and in the Northern Hemisphere at the Arctic station NyÅlesund, on the island of Spitsbergen [Asheim, 1983]. Properties of SE were discussed and refined in [Guglielmi, Dovbnya, 1973, 1974a,b; Guglielmi, 1979; Troitskaya, 1979; Fraser-Smith, 1982; Asheim, 1983; Morris, Cole, 1986, 1987; Guglielmi, Pokhotelov, 1996]. One of the newly discovered SE features is that the SE spectrum contains a stable 5-minute carrier modulation lasting a few hours [Guglielmi et al., 2015]. Such an example is shown in Figure 1. 
The coincidence of the period of the frequency modulation found in the SE spectrum with the 5-minute period common to fluctuations of the solar photosphere is of great interest because it most likely indicates the existence of a genetic relation between the frequency modulation of the emission and pulsations of the solar surface.

In this paper, we examine in more detail spectral features of SE as observed at the observatory Vostok in 1966-1971.

\section{DATA}

We have used archival data with magnetic tape recordings of ULF oscillations. Observations were made at the Antarctic station Vostok (geomagnetic coordinates: $\Phi=-89.3^{\circ}$; longitude $\Lambda=139.5^{\circ}$ ) from 1966 to 1971 . For further analysis, we digitized the magnetic recordings and built up spectrograms that reflected information about the dynamics of spectral composition of a signal in the range $0-5 \mathrm{~Hz}$ in frequency-time coordinates. We also utilized the $K_{\mathrm{p}}$ and $A_{\mathrm{p}}$ indices to analyze geomagnetic conditions.

\section{RESULTS}

In the SE spectrum, the 5-minute modulation is most pronounced. Frequency fluctuations of this period are present in about $70 \%$ of the total SE observation time. They can be observed both in their "pure" form and simultaneously with fluctuations of other periods. Figure 2 illustrates a 12-hour SE recording with a frequency modulation period close to 5 minutes.

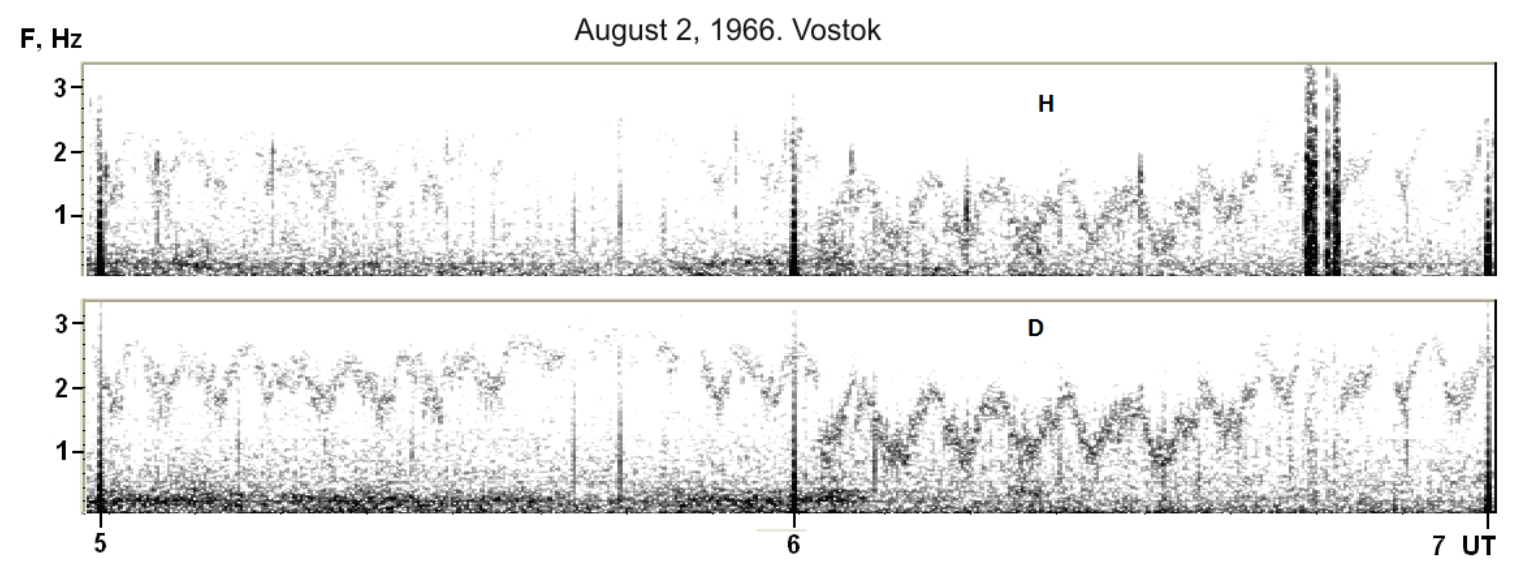

Figure 1. Dynamic spectrum of $H$ and D SE components with 5-minute modulation of carrier frequency

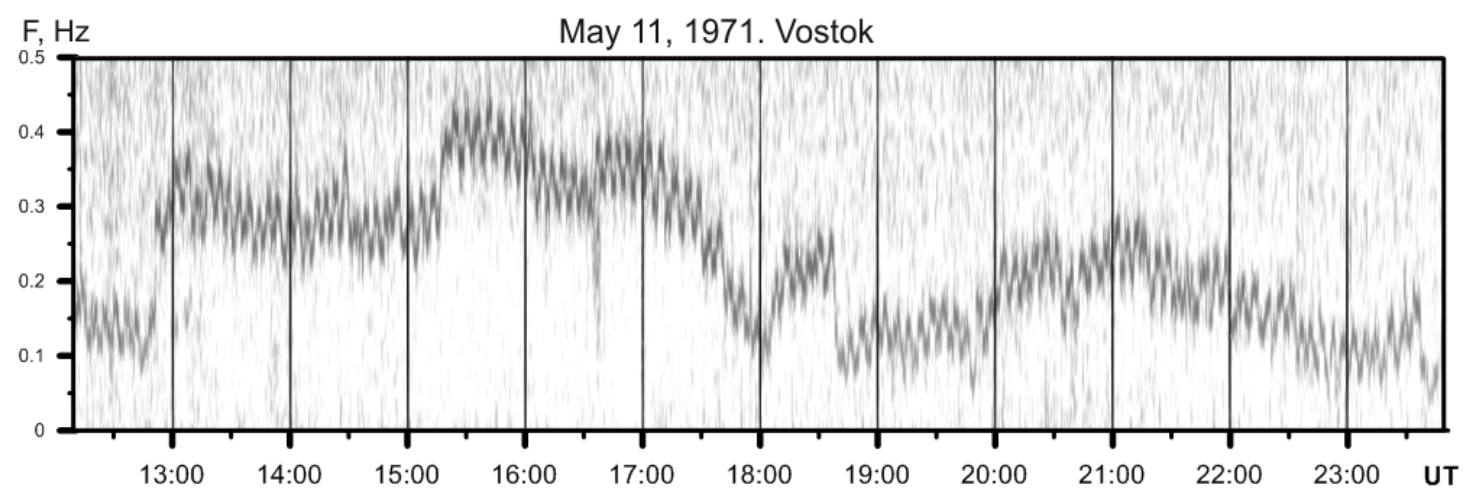

Figure 2. Serpentine emission with 5-minute frequency modulation 
Per-pixel spectrogram processing provides a discrete set of values of the curve that describes an SE frequency variation. Then, this curve is used to find relative signal power spectral density. An example of the spectrum is given in Figure 3. By comparison, Figure 4 shows the spectrum of solar surface oscillations obtained from the Doppler analysis of intensity of the light flux integrated over the solar surface. It is seen from this figure that peaks of spectral densities coincide, and, moreover, bandwidths of SE spectra and solar surface oscillations differ slightly.

One thing is noteworthy. To achieve the desired resolution of the spectrum of solar frequency fluctuations and suppress solar noise in observations, signals are analyzed coherently for several months [Christensen-Dalsgaard, 2002], while SE observations would enable us to track solar surface oscillations in near real-time.

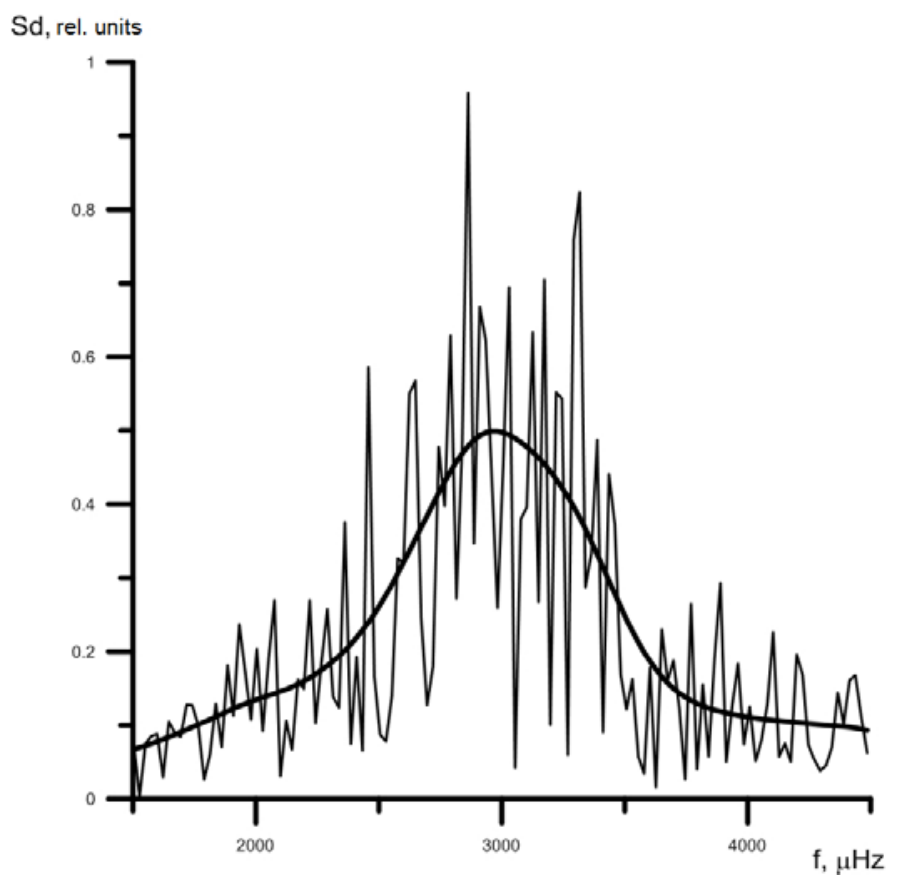

Figure 3. The power spectrum of frequency fluctuations for the serpentine emission event of May 11, 1971

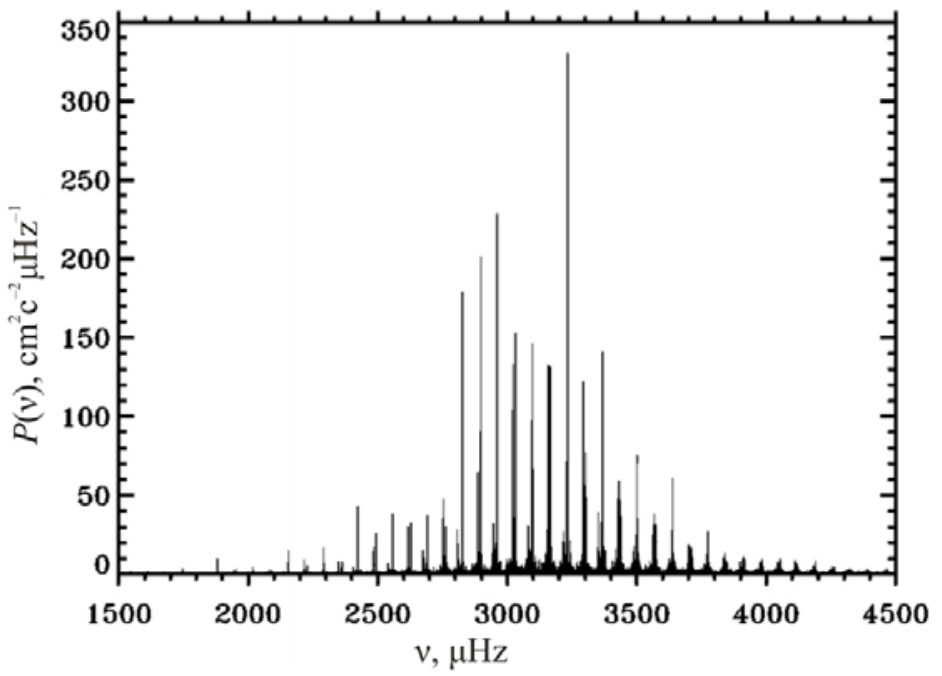

Figure 4. Power spectrum of solar oscillations as inferred from Doppler observations of light intensity integrated over the solar disk. The data are from six stations and cover about four months of observations (see [Elsworth et al., 1995; Christensen-Dalsgaard, 2002]) 


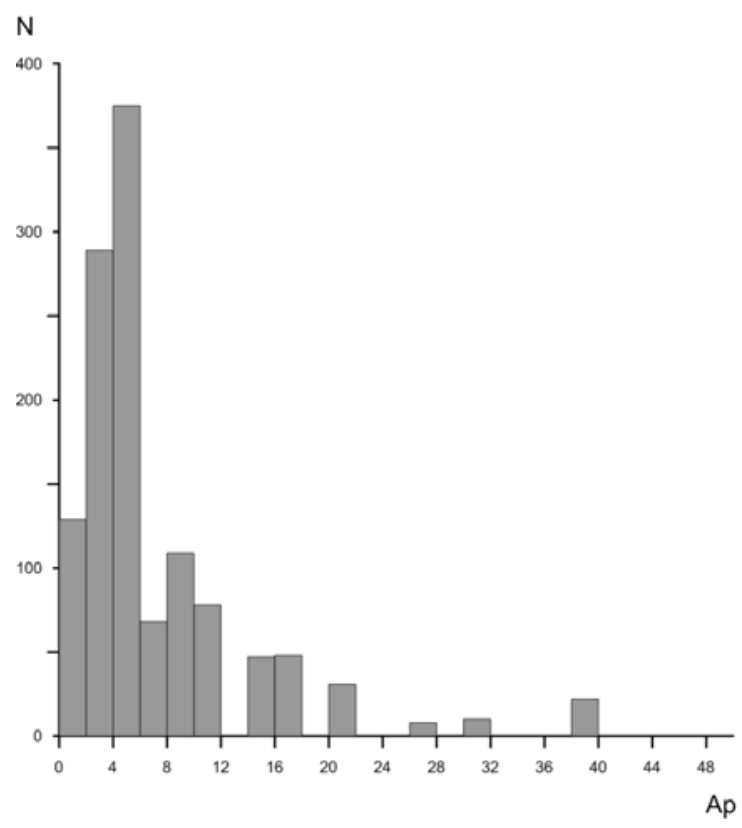

Figure 5. Histogram of distribution of the number of SE one-hour time intervals as a function of the $A_{\mathrm{p}}$ index

It is interesting to find out how magnetospheric disturbances affect the probability of observation of SE pulsations. To do this, we have analyzed three months of continuous recordings of the magnetic field at the observatory Vostok from April to June 1971. Within this time period, 1284 hour intervals contain serpentine emission with the 5-minute modulation of carrier frequency. Figure 5 presents a histogram of distribution of SE time intervals as a function of the $A_{p}$ index, which is linearly dependent on the level of magnetospheric disturbance.

In most cases, SE with 5-minute modulation occurs approximately in $70 \%$ of the total SE observation time under moderately quiet geomagnetic conditions $\left(A_{\mathrm{p}}=0-6\right.$, and thus $\left.K_{\mathrm{p}}=0-2\right)$.

The spectrum of solar surface oscillations contains information about processes in the differentially rotating solar convection zone above the tachocline. There also exist other features that might not be reflected in SE frequency pulsations, but nevertheless there is a close connection between processes on the Sun, such as proton flares, and dynamic SE spectrum variations [Dovbnya et al., 1994]. Furthermore, as inferred from experimental observations of Alfvén waves in near-Earth interplanetary space, there are 5-minute oscillations in their spectrum, which are clearly associated with 5-minute solar oscillations [Potapov et al., 2013]. The question about transformation of 5-minute solar surface oscillations into Alfvén wave energy and their possible contribution to the spectrum of frequency modulation of the serpentine emission has been discussed in detail in [Guglielmi et al., 2015].

\section{CONCLUSIONS}

Our analysis shows the following.

1. The 5-minute frequency modulation prevails in the spectrum of SE frequency variations.

2. Oscillations with this period of frequency modulation are recorded approximately in $70 \%$ of the total SE observation time under moderately quiet geomagnetic conditions $\left(K_{\mathrm{p}}=0-2\right)$. 
3. The power spectrum of SE frequency modulation with a peak at periods close to 5 minutes is similar to the power spectrum of 5-min solar oscillations.

Thus, it is clear that SE fluctuations reflect the dynamics of large-scale structures on the solar surface and can be an effective tool for studying helioseismological problems.

This work was supported by RFBR grants No. 16-05-00631 and 16-05-00056.

\section{REFERENCES}

Asheim S. Serpentine emissions in the polar magnetic field. Oslo, 1983, 8 p. (Rep. Ser. No. 83-38, Inst. of Physics).

Christensen-Dalsgaard, J. Helioseismology. Rev. Mod. Phys. 2002, vol. 74, pp. 1073-1129. DOI: 10.1103/RevModPhys.74.1073.

Dovbnya B.V., Zotov O.D., Klain B.I., Kurazhovskaya N.A. Dynamics of the SE-type emission before intense proton flares on the Sun. Geomagnetism and Aeronomy. 1994, vol. 34, no. 3, pp. 419-421.

Elsworth Y., Howe R., Isaak G.R., McLeod C.P., Miller B.A., van der Raay H.B., Wheeler S.J., New R. Performance of the BISON network in 1981-present. : Helio- and Astero-Seismology from Earth and Space (GONG'94): Proc. P. 392-397. (ASP Conference Ser. No. 76 / Eds. R.K. Ulrich, E.J. Rhodes, Jr., and W. Dappen). San Francisco, 1995.

Fraser-Smith A.C. ULF/lower-ELF electromagnetic field measurements in the polar caps. Rev. Geophys. Space Phys. 1982 , vol. 20, pp. 497-512. DOI: 10.1029 / RG020i003p00497.

Guglielmi A.V. MGD-volny v okolozemnoi plazme [MHD Waves in the Near-Earth Plasma]. Moscow, Nauka Publ., 1979, 139 p. (In Russian).

Guglielmi A.V., Dovbnya B.V. Hydromagnetic emission of the interplanetary plasma. Pis'ma $v$ ZhETF [JETP Letters]. 1973, vol. 18, iss. 10, pp. 601-604. (In Russian).

Guglielmi A.V., Dovbnya B.V. Hydromagnetic emission of the interplanetary plasma. Astrophys. Space Sci. 1974a, vol. 31, pp. 11-29.

Guglielmi A.V., Dovbnya B.V. Observations of geomagnetic pulsations in the range of 0-2 $\mathrm{Hz}$ with deep modulation of the carrying frequency. Geomagnetizm i aeronomiya [Geomagnetism and Aeronomy]. 1974b, vol. 14, no. 5, pp. 868-870. (In Russian).

Guglielmi A.V., Pokhotelov O.A. Geoelectromagnetic Waves. Bristol, Philadelphia, IOP Publ. Ltd., 1996. 402 p.

Guglielmi A., Potapov A., Dovbnya B. Five-minute solar oscillations and ion-cyclotron waves in the solar wind. Solar Phys. 2015, vol. 290, no. 10, pp. 3023-3032. DOI: 10.1007 / s11207-015-0772-2.

Morris R.J., Cole K.D. "Serpentine emission" at the high latitude station Davis (17-23 September 1981). Exploration Geophys. 1986, vol. 17, p. 15. DOI: 10.1071 / EG986015.

Morris R.J., Cole K.D. "Serpentine emission" at the high latitude Antarctic station, Davis. Planet. Space Sci. 1987, vol. 35, pp. 313-328. DOI: 10.1016/0032-0633(87)90158-9.

Potapov A.S., Polyushkina T.N., Pulyaev V.A. Observations of ULF waves in the solar corona and in the solar wind at the Earth's orbit. J. Atmos. Solar-Terrestrial Phys. 2013, vol. 102, pp. 235-242. DOI: 10.1016/j.jastp.2013.06.001.

Troitskaya V.A. Geomagnetic pulsations in the polar cap. International Workshop on Selected Topics of Magnetospheric Physics. Magnetospheric Study: Proc. Japanese IMS Comm., Tokyo, 1979, pp. 121-123. 